\section{NEUROPSYCHOLOGICAL EVALUATION ON THREE SUBTYPES OF SCHIZOPHRENIA}

\author{
H. Hwu, ${ }^{*}$ M. S. Hua, C. H. Chen, S. K. Liu, T. J. Hwang, \\ C. M. Liu, M. H. Hsieh \\ Department of Psychiatry, National Taiwan University Hospital, \\ Taipei, Taiwan
}

Purpose: Based on the psychopathological manifestation and treatment response, this study intends to delineate schizophrenia into different valid subtypes for facilitating the etiological and genetic studies. Methods: One hundred and sixty three consecutively admitted patients of schizophrenia were prospectively followed up for 2 years and serial psychopathological assessments were done using Positive and Negative Syndrome scale (PANSS). The data of PANSS assessed at 6-month after admission for inpatient treatment were analyzed using the generalized association plot to cluster patients. For a validity study on these subtypes, neuropsychological functions were examined including sustained attention measured by continuous performance test (CPT), WAIS-R, WMS$\mathrm{R}$ and Trial A, Trial B. the CPT was done during index admission and at 2-4 year follow-up; all other tests were examined at 2-4 year follow-up. Results: Three subtypes of schizophrenia could be delineated among 163 cases. These three subtypes were found to be valid with clinical characteristics: The remitted group (RMG) [61cases, $37.4 \%$ ] was defined to have only minimal degree of symptoms after neuroleptic treatment, the marked blunt group (MBD) [50 cases, 30.7\%] was defined to have only marked negative symptoms, which persisted through the follow-up course; the group with persistent delusion/ hallucination (PDHG) [52 cases, $31.9 \%$ ] was defined by having a persistence of delusion and hallucination symptoms through the follow-up course, youngest age of onset and poor treatment response. The MBG had the worst CPT as compared with other two groups $(\mathrm{F}=5.54, \mathrm{df}=2, \mathrm{P}=0.005)$. The difference among three groups in arithmetic $(\mathrm{F}=2.87, \mathrm{df}=2$, $\mathrm{P}=0.0615)$, information $(\mathrm{F}=3.09, \mathrm{df}=2, \mathrm{P}=0.05)$, similarity $(F=3.11, d f=2, P=0.0492)$, VIQ $(F=3.86, d f=2, P=0.0249)$, verbal paired test $(\mathrm{F}=6.38, \mathrm{df}=2, \mathrm{P}=0.0026)$, trail $\mathrm{A}(\mathrm{F}=4.22, \mathrm{df}=2$, $\mathrm{P}=0.0177)$, and trial $\mathrm{B}(\mathrm{F}=4.93$, df $2, \mathrm{P}=0.0096)$ were mainly contributed by the worst performance of the PDHG among these three subtypes. The differences in test scores between the RMG and MBG were not significant in these variables. Conclusion: The separation of schizophrenia into three subtypes was found to be valid. The PDHG was hypothesized to be the neurodevelopment subtype. Impaired CPT differentiates the RMG and BMG. The BMG could be a neurodegenerative subtype and the RMG a subtype of dopamine psychosis.

\section{EPOS - GENERAL OUTLINES OF THE "EUROPEAN PREDICTION OF PSYCHOSIS STUDY"}

J. Klosterkötter,* M. Birchwood, M. Hambrecht, D. Linszen, H. Reventlow, S. Ruhrmann, R. K. Salokangas, J. L. Vàzquez-Barquero, \& EPOS Group Department of Psychiatry and Psychotherapy, University of Cologne, Cologne, NRW, Germany

The European Prediction of Psychosis Study (EPOS) marks the first European multi-centre effort on early recognition and intervention for persons at risk for psychosis. Participants are Cologne, Germany; Turku, Finland; Amsterdam, The Netherlands; Santander, Spain; Birmingham, UK. This EC-funded study will provide a unique data set on persons of high risk for psychosis in different European countries and health systems. Besides a multi-level assessment including psychopathology, neuropsychology, neurophysiology, and brain imaging, this prospective, longitudinal field-study also monitors the course of disabilities, quality of life, and interventions, e.g. psychological and pharmacological treatments, in prodromal persons. In addition, EPOS collects retrospective data on pathways to care and obstacles for adaequate treatment. During a 24-months recruiting period, each of the 5 collaborating centres includes at least 50 prodromal persons. The presence of transient or attenuated psychotic symptoms, a familial risk for psychosis plus decline in functioning, or subtle self-perceived cognitive and perceptual deficiencies define the at-risk state. The outcome is measured at 9- and 18-months follow-ups in order to establish a "risk profile". A comprehensive overview on the general outlines of EPOS, as well as major developments in the first year of the study will be presented.

\section{HOW FAR IS PSYCHOPATHOLOGY STILL MEANINGFUL FOR EARLY DETECTION OF SCHIZOPHRENIA?}

\section{Lanzaro \\ Private Job, San Gennarello di Ottaviano NAPLES, Napoli, Italy}

In this study we have tried to summarize current limitations and future perspectives of the diagnostic concept of prodrome in schizophrenia, and their implications for research, clinical practice and primary care. First of all, the essence of DSM-IV concept of what should be considered prodromic and its operative consequences raises some problems. In fact, it mentions a cluster of five symptoms, listed as examples, which are merely residua of DSM-III and DSM-III-R operational criteria for the schizophrenia prodrome. Many of the previous operational criteria have been dropped from DSM-IV because of their poor relibility and validity, but it globally emerges that the attempt of simplification of the previous classification led DSM.-IV to an inconsistent conceptualization of the prodromal phases of schizophrenia. As in clinical medicine, the word prodrome always underlies a retrospective concept: it denotes a cluster of features with a temporal but not a causal longitudinal relationship with the development of schizophrenia. Because prodromal symptoms can resolve, this means that they do not guarantee transition to a first psychotic episode, but may instead indicate an increased risk of this transition. This is why other authors (Mc Gorry) proposed to label a specific cluster of early psychopathological changes as an at risk mental state. This term highlighs the idea that the syndrome confers risk for the development of subsequent psychosis, but that psychosis is not inevitable: it obviously emerges the problem of false-positive predictions. In any case, we do use this terms because specific prospective predictors do not currently exist. But it is crucial to note that wheter any such risk factors were ever clearly identified, they would be best conceptualized as early phenomena of the disorder itself. We propose a re-examination of the traditional prodrome concept trough prospective studies which consider the early phases of pshycoses as a dimensional phoenomenological process, rather than a categorical list of nonspecific symptoms at any one point. An early detection classification system for primary care will need to be characterized by simplicity; on the other hand, a renaissance of psychopatology in the psychiatric research focused on the above issues should be in our opinion, encouraged. 